\title{
Four colour photometry of late-type binary systems
}

\section{New light curves of BH Virginis ${ }^{\star, \star \star}$}

\author{
R. Clement ${ }^{1}$, M. Garcia ${ }^{2}$, V. Reglero ${ }^{1}$, J.V. Clausen ${ }^{3}$, A. Bravo ${ }^{2}$, J. Suso ${ }^{1}$, and J. Fabregat ${ }^{1}$ \\ 1 Departamento de Astronomía y Astrofísica, Universidad de Valencia, Dr Moliner 50, E-46100, Burjassot, Valencia, Spain \\ 2 Departamento de Física Aplicada, E.U.I.T. Industrial, Universidad Politécnica de Madrid, Ronda de Valencia 3, E-28012 \\ Madrid, Spain \\ 3 The Niels Bohr Institute for Astronomy, Physics and Geophysics, Astronomical Observatory, Brorfeldevej 23, DK-4340 Töllöse, \\ Denmark
}

Received June 21; accepted August 5, 1996

\begin{abstract}
This paper presents new complete uvby light curves of the late-type detached eclipsing binary BH Vir $(\mathrm{G} 0 \mathrm{~V}+\mathrm{G} 5 \mathrm{~V}, P=0 \mathrm{~d} 81687099)$.

This binary system has been observed during four campaigns at the Calar Alto Observatory (Almeria, Spain) and at the European Southern Observatory (La Silla, Chile). This observations are part of a 6 year uvby and $\mathrm{H} \beta$ monitoring program of low mass eclipsing binaries which main objective is to provide accurate absolute astrophysical parameters for late-type main sequence stars. 1585 points spreaded over the binary period and covering both eclipses are given. The internal accuracy of the standard photometry measured as the mean RMS of the differences between standard value and observed value for the standard stars observed along the program is quoted in a few millimagnitudes. Detailed analysis of BH Vir, based on these light curves, will be published separately.
\end{abstract}

Key words: stars: binaries: eclipsing — stars: individual: $\mathrm{BH}$ Vir

\section{Introduction}

$\mathrm{BH} \operatorname{Vir}(V=9.638, G 0 V+G 2 V, P=0 \mathrm{~d} 81687099)$, is a detached, main sequence, late type, double line eclipsing binary. In spite of lot of observing effort done up to now,

Send offprint requests to: $\mathrm{R}$. Clement

* Based on observations collected with the Danish $0.5 \mathrm{~m}$ telescope at the European Southern Observatory (ESO), La Silla, Chile and the Spanish $1.5 \mathrm{~m}$ telescope at Calar Alto, Almería, Spain.

** Table 2 will be accessible only in electronic form at the CDS via anonymous ftp to cdsarc.u-strasbg.fr (130.79.128.5) or via http://cdsweb.u-strasbg.fr/Abstract.html mass, radius, temperatures and abundances are poorly defined in the low mass range where only a few stars have astrophysical parameters calculated with the required accuracy for a detailed evolutionary modelling. With this aim, we have performed a 6 year monitoring program to obtained good photoelectric uvby and $\mathrm{H} \beta$ light curves for late-type binary systems, Clement et al. (1997). BH Vir was a good candidate and was thus included in our program of absolute parameters determination for late-type binary systems.

Photometric studies of the interesting eclipsing binary BH Vir have been carried out by several authors: Kitamura et al. (1957), Koch (1967), Sadik (1978), Botsula (1978), Hoffmann (1982), Guiricin et al. (1984), Scaltriti et al. (1985), and Derman et al. (1989). Recently in a detailed analysis of the available light curves using the Wilson-Devinney code, Zhai et al. (1990) derived the most relevant astrophysical parameters of both components. More recently, Vincent (1993), using surface imaging techniques includes BH Vir in the list of binary systems that show occultation of the secondary star by the primary star, that is, the secondary eclipse of BH Vir is total. The analysis of the photometric light curves for $\mathrm{BH}$ Vir is a difficult task mainly due to the large and very fast amplitude variations outside eclipses. Koch (1967) reported changes in the depth of the primary eclipse of a tenth of a magnitude, in only few days. Although we observed a variation of the same order between the last two campaigns, we found, however, no appreciable variations of the light curves within the short time interval of each campaign (weeks). Despite its showing intrinsic variability, BH Vir has been included in our program due to the lack of late-type eclipsing binaries without photometric variations outside eclipse. Relevant photometric elements can be extracted for such systems too, once the light curves have been cleaned of the activity waves attributed to the 
presence of spots in the stellar surface. To attain the maximum accuracy in the determination of the astrophysical parameters when analysing binary systems affected by activity, we have implemented a new iterative method to estimate more accurately the contribution of activity.

In this paper we present accurate and complete photoelectric uvby and $\mathrm{H} \beta$ light curves for $\mathrm{BH}$ Vir, whose light curve has been fully covered at least three times in four different epochs.

A complete analysis of BH Vir based on these data will be published separately.

Table 1. Standard photometry for BH Vir and the comparison stars

\begin{tabular}{|c|c|c|c|}
\hline & BH Vir & Comp. 1 & Comp. 2 \\
\hline \multirow{5}{*}{$\begin{array}{l}\text { HD no. } \\
\alpha_{2000} \\
\delta_{2000} \\
\text { Sp. type }\end{array}$} & HD121909 & HD121935 & HD139643 \\
\hline & $13^{\mathrm{h}} 58^{\mathrm{m}} 25^{\mathrm{s}}$ & $13^{\mathrm{h}} 58^{\mathrm{m}} 42^{\mathrm{s}}$ & $13^{\mathrm{h}} 56^{\mathrm{m}} 44^{1}$ \\
\hline & $-01^{\circ} 39^{\prime} 38^{\prime \prime}$ & $-01^{\circ} 37^{\prime} 00^{\prime \prime}$ & $-00^{\circ} 53^{\prime} 08^{\prime \prime}$ \\
\hline & G0V+G2V & & \\
\hline & 9.638 & 9.814 & 9.551 \\
\hline$V(0.25)$ & 12 & 13 & 14 \\
\hline \multirow{2}{*}{$V(0.00)$} & 10.517 & & \\
\hline & 17 & & \\
\hline \multirow[t]{2}{*}{$V(0.50)$} & 10.151 & & \\
\hline & 11 & & \\
\hline \multirow[t]{2}{*}{$(b-y)(0.25)$} & 0.384 & 0.291 & 0.379 \\
\hline & 9 & 9 & 7 \\
\hline \multirow[t]{2}{*}{$(b-y)(0.00)$} & 0.433 & & \\
\hline & 16 & & \\
\hline \multirow{2}{*}{$(b-y)(0.50)$} & 0.381 & & \\
\hline & 11 & & \\
\hline \multirow[t]{2}{*}{$m_{1}(0.25)$} & 0.191 & 0.159 & 0.175 \\
\hline & 18 & 14 & 11 \\
\hline$m_{1}(0.00)$ & 0.195 & & \\
\hline \multirow{2}{*}{$m_{1}(0.50)$} & 0.158 & & \\
\hline & 17 & & \\
\hline \multirow[t]{2}{*}{$c_{1}(0.25)$} & 0.308 & 0.494 & 0.332 \\
\hline & 24 & 20 & 21 \\
\hline \multirow[t]{2}{*}{$c_{1}(0.00)$} & 0.292 & & \\
\hline & 39 & & \\
\hline \multirow[t]{2}{*}{$c_{1}(0.50)$} & 0.324 & & \\
\hline & 22 & & \\
\hline \multirow[t]{2}{*}{$\beta(0.25)$} & 2.592 & 2.670 & 2.604 \\
\hline & 14 & 16 & 10 \\
\hline \multirow[t]{2}{*}{$\beta(0.00)$} & 2.570 & & \\
\hline & 12 & & \\
\hline \multirow[t]{2}{*}{$\beta(0.50)$} & 2.627 & & \\
\hline & 14 & & \\
\hline
\end{tabular}

\section{The observations}

Part of the data were taken in March 1990 and January 1992 at The Calar Alto Observatory (Almeria, Spain). The telescope used was the $1.5 \mathrm{~m}$ reflector equipped with either a multipurpose one-channel photoelectric photometer or, alternately, with a four-channel $u v b y-\mathrm{H} \beta$ spectrograph photometer, both using narrow and wide $\mathrm{H} \beta$ filters. The last two photometric campaigns were carried out in April 1993 and May 1994 at The European Southern

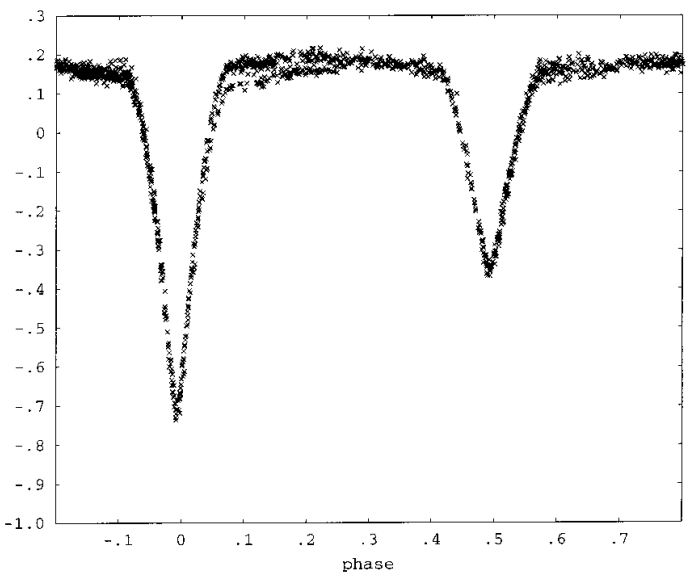

Fig. 1. BH Vir differential light curve. $y$ filter

Observatory (La Silla, Chile) using the $0.5 \mathrm{~m}$ Danish telescope equipped with the same $u v b y-\mathrm{H} \beta$ four-channel photometer, with Strömgren uvby filters as well as Crawford's narrow and wide $\mathrm{H} \beta$ filters.

The 1585 measurements of BH Vir were obtained in the Strömgren uvby system during four observing campaigns.

- four nights in March 1990 using the mono-channel photometer $U B V u v b y \mathrm{H} \beta$ mounted on the $1.5 \mathrm{~m}$ telescope of the Observatorio Astronómico Nacional at The Calar Alto Observatory at Almeria, Spain,

- six nights in January 1992 using a four-channel photometer $u v b y \mathrm{H} \beta$ mounted on the $1.5 \mathrm{~m}$ telescope of the Observatorio Astronómico Nacional at The Calar Alto Observatory at Almeria, Spain,

- eleven nights in April 1993 using a four-channel photometer identical to the one used in the previous campaign mounted on the $0.5 \mathrm{~m}$ Danish telescope at the European Southern Observatory (ESO), La Silla, Chile

- eight nights in May 1994 using the same four-channel photometer on the $0.5 \mathrm{~m}$ Danish telescope at the ESO, La Silla, Chile.

Details about the instrumental configuration and the reduction of the photometry can be found in Clement et al. (1997) and references included there.

HD 121935, $(V=9.67, F 4 V)$ and HD $139643(V=$ $9.44, G 0 V)$, were used as comparison and check stars. Their magnitudes and spectral type being similar to $\mathrm{BH}$ Vir itself. Average magnitudes and colour indices for the comparison stars are given in the last two columns of Table 1, with an indication of their accuracy measured through the RMS dispersion of the observed values. In Table 1 is also given averaged magnitudes and colour indices for $\mathrm{BH}$ Vir in the eclipses and first quadrature. The constancy of the comparison star was checked every night. The internal RMS errors for the 569 differences (comparison- check) 0.012, 0.010, 0.015, 0.021, 0.017 in magnitudes for $V,(b-y), m_{1}, c_{1}$ and $\beta$ respectively is of the same order as that obtained for main-program stars. 
Extinction corrections were applied based on nightly coefficients. The differential uvby light curves of $\mathrm{BH}$ Vir with respect to HD 121935 were calculated. Besides the program stars, a selected set of standard stars from the lists of Perry et al. (1987) and Olsen (1991), was measured during the best photometric nights in order to transform the instrumental magnitudes into the standard system, as the accuracy of our standard photometry is quoted in a few millimagnitudes (0.009 mag in the $y$ filter).

The 1585 differential magnitude (BH Vir - HD 121935) values in the standard system are given in Table 2. Figure 1 presents the $\mathrm{BH}$ Vir differential light curve in the $y$ filter in which the effect of activity is clearly visible.

Acknowledgements. The $1.5 \mathrm{~m}$ telescope at Calar Alto is operated by the Observatorio Astronomico Nacional. The $0.5 \mathrm{~m}$ telescope is operated by the Copenhagen Group. We want to express our gratitude to the observatories staff. This work has been supported by the Spanish Comision Interministerial de Ciencia y Tecnologia (PB90-0001-C02)

\section{References}

Botsula R.A., 1978, in: Varable Star (Russian) 20, 577

Clement R., Garcia M., Reglero V., et al., 1997, A\&AS 123, 1

Derman E., Akalin A., Demircan O., 1989, Inf. Bull. on Variable Stars, No. 3404

Guiricin G., Mardirossian F., Mezzetti M., 1984, MNRAS 206, 305

Hoffmann M., 1982, A\&A 471, 561

Kitamura M., Nakamura T., Takahashi C., 1957, PASP 9, 191

Koch R.H., 1967, AJ 72, 411

Olsen E.H., 1991, (private communication)

Perry C.L, Olsen E.H, Crawford D.L., 1987, PASP 99, 1184

Sadik A.R., 1978, Phd Thesis, U. Manchester

Scaltriti F., Cellino A., Busso M., 1985, A\&A 149, 11

Vincent A., Piskunov N.E., Tuominen I., 1993, A\&A 278, 523

Zhai D.S., Qiao G.J., Zhang X.Y., 1990, A\&A 237, 148 